\title{
Localization phenomenon investigation on Smated stainless samples by speckle interferometry
}

\author{
J. Petit, G. Montay, and M. François
}

LASMIS, Institut Charles Delaunay, UMR CNRS 6279,
University of Technology of Troyes, 10010 Troyes, FRANCE

\begin{abstract}
In-plane Electronic Speckle Pattern Interferometry (ESPI) has been successfully used during tensile tests on SMA-treated (Surface Mechanical Attrition Treatment) and as received 316L stainless steel in order to measure the strain rate field. The heterogeneity in the strain rate field can be observed from a stage of deformation which doesn't coincide with the classic Considère's criterion $(\mathrm{dF}=0)$ for the diffuse neck initiation (or plastic instability). The initiation of this heterogeneity strongly depends on treatments undergone by the material. In this paper, a comparison was performed about the heterogeneity initiation of specimens SMATed during 30 minutes with $2 \mathrm{~mm}$ shot, $3 \mathrm{~mm}$ shot and a non-treated sample. The formation of the two slip band system until fracture of the tensile specimen was also studied.
\end{abstract}

\section{Introduction}

$316 \mathrm{~L}$ austenitic stainless steel is a widely used engineering material due to its excellent corrosion and oxidation resistance and good formability. However, application of this material is hindered by its low mechanical strength and poor anti-friction properties. Strengthening of austenitic stainless steels has drawn much attention in the past decades and various approaches have been developed, such as varying its chemical compositions to induce solid solution hardening [1,2] or grain refinement [3-5].

Surface mechanical attrition treatment (SMAT) is a recently developed technique that can effectively induce grain refinement down to nanometre regime in the surface layer (of several tens of micrometers depth) of bulk materials [6-10]. The nanocrystalline layer is free of contamination and porosity because the nanocrystallization process is induced by severe plastic deformation at very high strain rates [7]. The grain size of the processed surface layer can be smaller than $10 \mathrm{~nm}$ in some materials.

In order to study mechanical behaviours of materials during tensile test, optical methods are more and more used, because they give a strain map on relatively large areas. Tensile test allows determining many mechanical properties of materials, such as Young's modulus, yield stress, ultimate tensile stress, strain hardening coefficient, ductility, etc... For most metals, at the beginning of the tensile test, strains are homogeneous along the specimen. Classically, it is considered [11] that 
the diffuse neck occurs when the force reaches its maximum $(\mathrm{dF}=0)$. Strains become heterogeneous in the whole specimen and then a two shear bands system appears with the localized neck.

Many optical methods exist, and among them interferometry and digital image correlation are suitable for our study. The latter is still today less sensitive than interferometric methods. Moreover, it doesn't allow following deformation increments in real time. ESPI (Electronic Speckle Pattern Interferometry) was consequently chosen during tensile test experiments. Complete theoretical and experimental description of ESPI can be found in $[12,13]$. This technique was widely used to study deformation localization in metals. By example, $[14,15]$ analysed homogeneous plastic deformation and localized neck during tensile test. [16] looked at the Portevin - Le Châtelier phenomenon. ESPI allows the temporal and spatial evolution of the displacement in the whole tested area of specimen to be followed with a high resolution in time and in space. Temporal phase shifting technique [17] was used to obtain fringes representative of the material displacement. This technique enables to get the phase of the interference pattern at all pixels of the image.

The aim of the present paper is to measure strain rate field by ESPI, as a relevant physical quantity to investigate the localization onset, and especially to make a comparison between three specimens, two specimens SMATed during 30 minutes with $2 \mathrm{~mm}$ and $3 \mathrm{~mm}$ shot, and one nontreated sample. The results of ESPI are compared to the classic Considère's criterion, and the development of the localization process was explored until fracture. Strain rate is then connected to the hardening effects induced by SMAT on the surface layers.

\section{Experimental procedures}

\subsection{Specimens}

Three specimens, Figure 1, were cut in a $1 \mathrm{~mm}$ thick sheet of commercial $316 \mathrm{~L}$ face-centered cubic (fcc) austenitic stainless steel. The roughness of the as received material is $\mathrm{R}_{\mathrm{a}} / \mathrm{R}_{\mathrm{t}} \approx 0.31 / 3.09 \mu \mathrm{m}$. The initial microstructure of the as received material is characterised by a grain size ranging between 10 and $60 \mu \mathrm{m}$. Two of the 316L specimens were SMATed on their two sides, as shown on Figure 1. The setup of SMAT processing can be found in previous publications $[6,7,9]$. The basic principle of treatment is the generation of plastic deformation in the top surface layer of a bulk material by means of a large number of repeated multidirectional and highly random impacts of spherical shot onto the sample surface (Figure 2). The induced plastic deformation in the surface layer with a large strain and a high strain rate results in a progressive refinement of coarse grains into the nanometre regime. Consequently, a nanocrystalline layer, approximately $20 \mu \mathrm{m}$ thick [18], was formed after SMAT. Underneath the nanocrystalline layer, the grain sizes increase gradually from the nanometre to the micrometre scales.

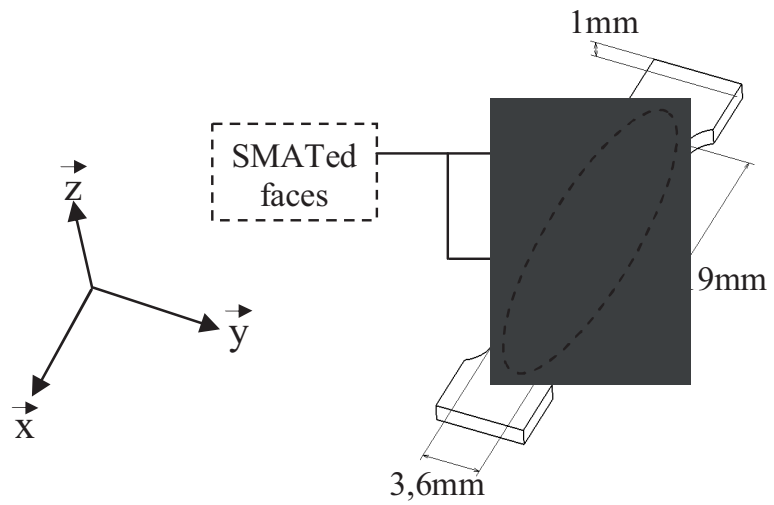

Fig. 1. Scheme of the specimen. 
The main parameters of SMAT processing used in this present work are as follow:

- vibration frequency of the chamber: $20 \mathrm{kHz}$,

- spherical steel shot (material 100Cr6) diameter: 2 and $3 \mathrm{~mm}$,

- treatment duration: 30 minutes,

- treatment conditions: in air at room temperature.

The roughness reached after SMAT is high enough, $\mathrm{R}_{\mathrm{a}} / \mathrm{R}_{\mathrm{t}} \approx 0.61 / 5.67 \mu \mathrm{m}$ for treatment with 2 mm diameter shot and $\mathrm{R}_{\mathrm{a}} / \mathrm{R}_{\mathrm{t}} \approx 0,73 / 5,49 \mu \mathrm{m}$ for treatment with $3 \mathrm{~mm}$ diameter shot.

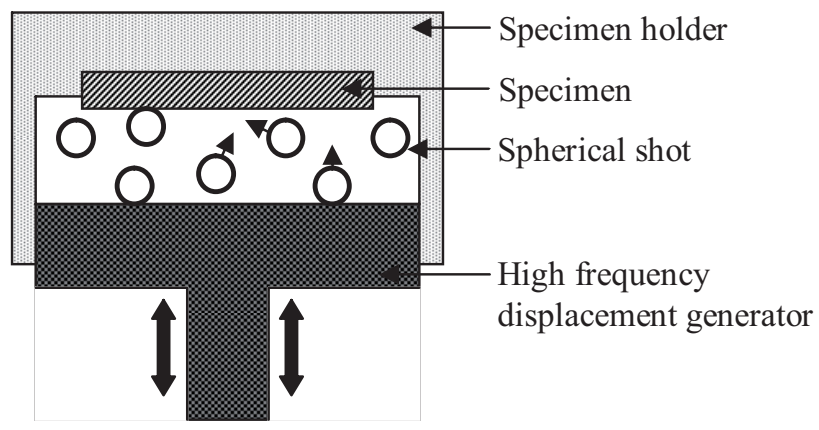

Fig. 2. Schematic illustration of the ultrasonic shot peening treatment set-up.

\subsection{Experimental setup}

The tests were performed on a $5 \mathrm{kN}$ capacity micro-tensile machine at a crossbeam speed varying from $5 \mu \mathrm{m} / \mathrm{s}$ at the beginning of experiments to $0.1 \mu \mathrm{m} / \mathrm{s}$ at the end. The surfaces of the specimens were left as it is for experiments. The experimental setup designed for strain rate measurements and mainly including the speckle interferometer and the tensile stage, is presented in Figure 3 . The wavelength of the He-Ne laser was $\lambda=632.8 \mathrm{~nm}$. The beam was separated into two beams by a beam-splitter and directed towards the specimen by mirrors. Between the mirrors and the specimen, each beam was expanded by a lens and filtered through a $15 \mu \mathrm{m}$ diameter pinhole. The present setup is an in-plane sensitive configuration, known as a Leendertz set-up, with a sensitivity vector $\vec{s}$ parallel to the tensile direction. The sensitivity vector is defined by $\vec{s}=\vec{n}_{1}-\vec{n}_{2}$, where $\vec{n}_{1}$ and $\vec{n}_{2}$ are the directing vectors of the two incoming beams $[12,13]$.

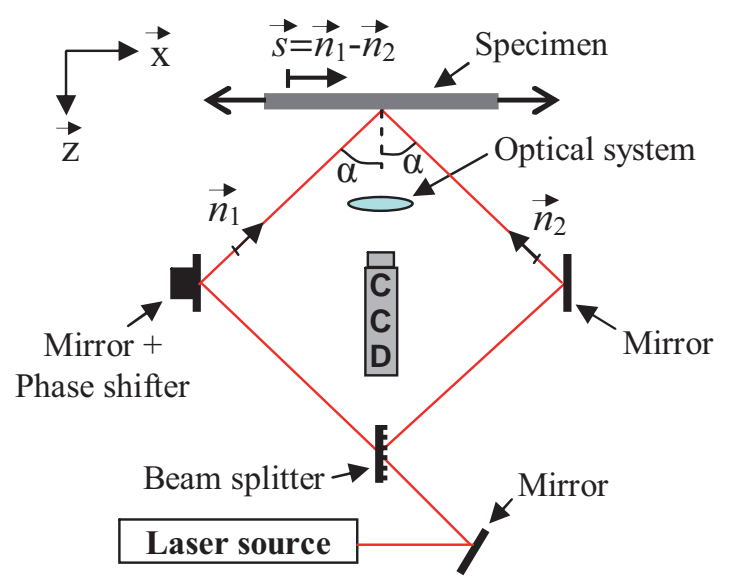

Fig. 3. Experimental setup: in-plane and micro-tensile machine. 


\subsection{Strain rate definition}

Before deformation, the intensity of light, $I_{R}$, in any point of the camera is given by:

$I_{R}(x, y)=I_{0}(x, y)(1+\gamma(x, y) \cos [\phi(x, y)])$,

where $I_{0}$ is the unknown average intensity, $\gamma$ is the visibility factor of the fringes and $\phi(x, y)$ is the phase distribution.

When a displacement $\vec{d}=d_{1} \vec{x}+d_{2} \vec{y}+d_{3} \vec{z}$ occurs, it produces a phase difference $\Delta \varphi(x, y)[12]$ :

$\Delta \varphi(x, y)=\frac{4 \pi}{\lambda} d_{1} \sin \alpha$,

where $d_{1}$ is the component of $\vec{d}$ parallel to $\vec{s}$ and $\alpha$ is the incidence angle of the laser beams (Figure 3). The intensity, $I$, after deformation becomes:

$I(x, y)=I_{0}(x, y)(1+\gamma(x, y) \cos [\phi(x, y)+\Delta \varphi(x, y)])$.

The difference between the two intensities $I$ and $I_{R}$, gives rise to a system of fringes and the resultant intensity is:

$\left|I(x, y)-I_{R}(x, y)\right|=2 I_{0}(x, y) \gamma(x, y)\left|\sin \left[\phi(x, y)+\frac{\Delta \varphi(x, y)}{2}\right] \sin \left[\frac{\Delta \varphi(x, y)}{2}\right]\right|$.

If $\Delta \varphi(x, y)=2 \pi$, then dark fringes arise and Eq. (2) becomes:

$d_{1}=\frac{\lambda}{2 \sin \alpha}$

where $s$ is the sensitivity of the interferometer expressed in $\mu \mathrm{m} /$ fringe. In the present case, the incidence angle is about $45^{\circ}$, so the sensitivity is $0.447 \mu \mathrm{m} /$ fringe.

The relative displacement between two points $\mathrm{A}$ and $\mathrm{B}$ is then:

$u_{A B}=N_{A B} \cdot S$,

where $N_{\mathrm{AB}}$ is the number of fringes (not necessarily an integer) between $\mathrm{A}$ and B. $N_{A B}$ is obtained after fringe unwrapping. The average strain between the two points can be expressed as:

$\varepsilon_{A B}=\frac{u_{A B}}{l_{A B}}$,

where $l_{\mathrm{AB}}$ is the distance between the two points and the mean strain rate is:

$\dot{\varepsilon}_{A B}=\frac{\varepsilon_{A B}}{\Delta t}$,

where $\Delta t$ is the time elapsed between the two pictures.

Crossbeam speed is implicitly included in the number of fringes $N_{\mathrm{AB}}$, it is consequently necessary to express strain rate in respect with a reference speed, $v_{0}$, arbitrarily chosen. Thus, strain rate becomes:

$\dot{\varepsilon}_{A B}=\frac{\varepsilon_{A B} \cdot v_{0}}{\Delta t \cdot v_{t}}$

where $v_{t}$ is the crossbeam speed when measurement is being done.

\subsection{Data recording}

Phase images were obtained by a CCD camera (1280 pixels $\times 980$ pixels, monochromatic, 8 digits) and saved on a computer. The temporal phase shifting was performed with a mirror mounted on a piezoelectric actuator. This technique gives immediately access to the phase difference $\Delta \varphi(x, y)$ in Equation (4). A resolution of nearly $1 / 20$ of a fringe can be reached. 
Being aware that the deformation is temporally varying, an uncertainty study using $N$-Bucket algorithm with $N=4$ and taking into account a crossbeam speed of $0.1 \mu \mathrm{m} / \mathrm{s}$ led to an error less than $3 \%$ on real displacement when $\Delta \varphi(x, y) \in[0,2 \pi]$ and less than $0.4 \%$ when $\Delta \varphi(x, y)>2 \pi$.

A long working distance microscope objective was placed in front of the CCD (Power: $4 \times$, Numerical Aperture: 0.12 , Focal Length: $30.8 \mathrm{~mm}$ ), in order to get an area observed by the camera approximately equal to $5 \mathrm{~mm} \times 4 \mathrm{~mm}$, like in [19], appropriate to study strain rate evolution of the specimens.

At regular time intervals during the experiment, white light images of the specimen were recorded on another computer, in order to get the total strain from a grid marked on the specimen.

\section{Results and discussion}

\subsection{Tensile curves}

Firstly, in Figure 4, the tensile curves of the three studied specimens are shown:

- (1) as received 316L stainless steel,

- (2) SMATed 316L stainless steel during $30 \mathrm{~min}$ with $2 \mathrm{~mm}$ diameter shot,

- (3) SMATed 316L stainless steel during $30 \mathrm{~min}$ with $3 \mathrm{~mm}$ diameter shot.

It can be noticed that the higher the tensile strength the smaller the fracture strain. It is worth mentioning that the yield stress of the material is almost doubled by the SMAT treatment while retaining significant ductility $(37 \%)$.

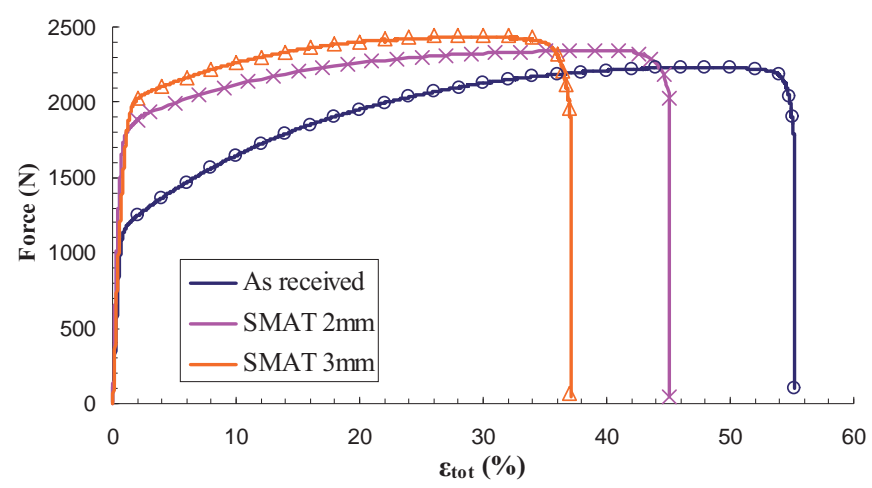

Fig. 4. Tensile curves for three states of the material.

\subsection{Time evolution of the strain rate and onset of localization}

In accordance with the results of [19-20], although it's about a different material, from the beginning of experiments to a certain point, the strain rate is homogeneous in the whole material. After this point, which occurs after the diffuse neck predicted by Considère's criterion, fringe pattern is no more a succession of fringes parallel between them, equidistant and perpendicular to the tensile direction, see Figure 5, consequently strain rate becomes heterogeneous.

On this figure, $\varepsilon_{\text {tot }}$ represents the total (longitudinal) strain when pictures are taken. $\varepsilon_{\text {tot }}$ was computed with the following equation:

$\varepsilon_{\text {tot }}=\ln \frac{\text { measured length }}{\text { initial length }}$. 
The measured length is obtained from a second camera working in white light. This camera continuously observes the distance between two points drawn at each end of the specimen. In the upper right corner of each picture, " $\Delta \mathrm{t}$ " corresponds to the time interval elapsed between two phase pictures subtracted to get fringes pictures.

The scenario of fringes pattern evolution, shown Figure 5 for the $3 \mathrm{~mm}$ SMATed specimen from a few time before diffused neck initiation until fracture, immediately gives the one of the strain rate evolution. This evolution is the same for the two others specimens except for the strain at which the localization appears.

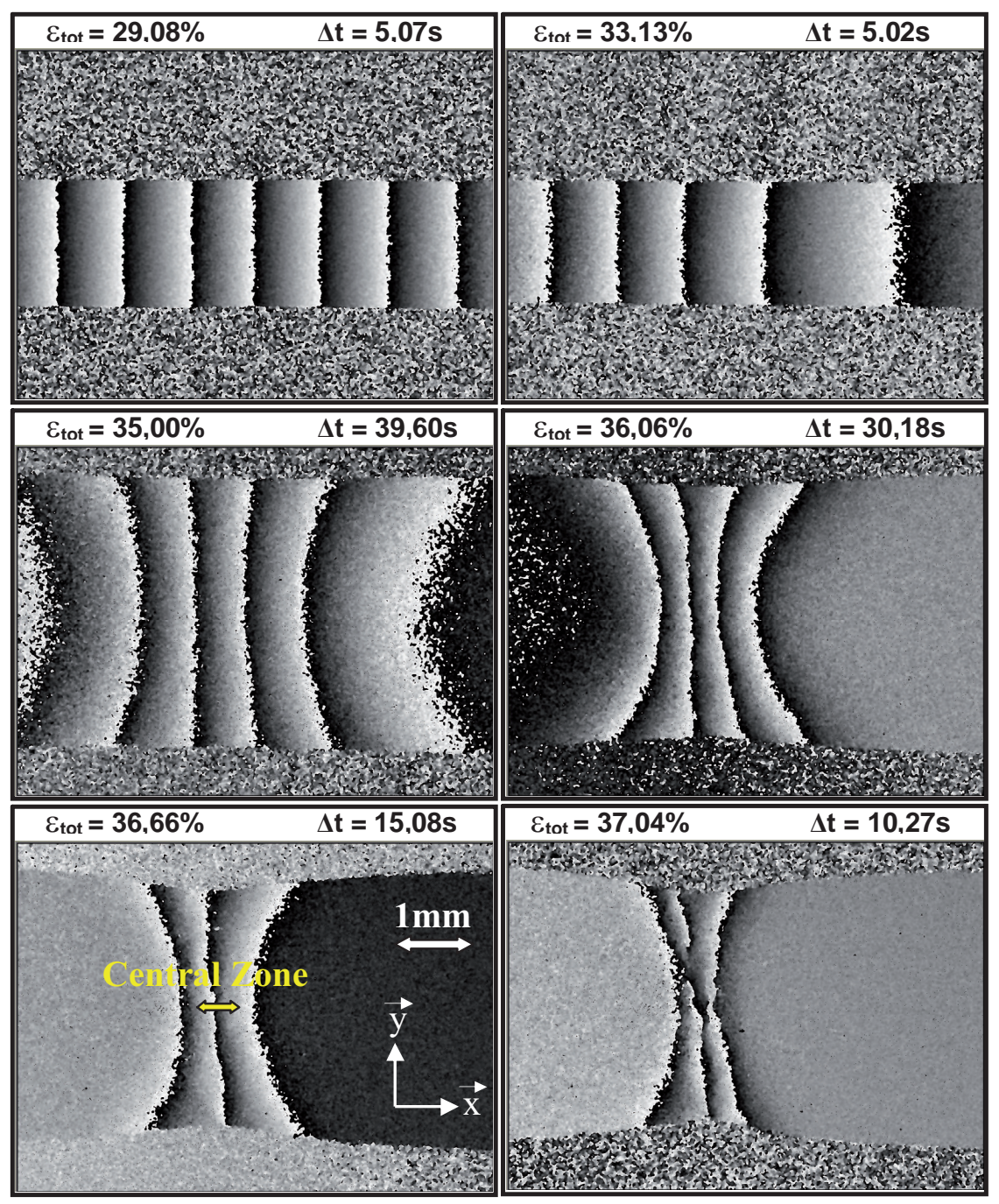

Fig. 5. Fringes pattern evolution and central zone where strain rate is evaluated. After $\varepsilon_{\mathrm{tot}}=33,20 \%$, magnification was increased on localization.

Strain rate evolution in central zone of the width of the specimens (Figure 5) is compared for the three specimens. The result is given Figure 6. The strain rate was calculated with Equation (9) at different stages of the tensile test and normalized in respect with tensile speed of $1 \mu \mathrm{m} / \mathrm{s}$. $\varepsilon_{\mathrm{AB}}$ in Equation (9) is determined from fringes patterns obtained by ESPI. 
It was mentioned before that, the more severe the treatment, the smaller the fracture strain and, as it can now be seen on Figure 6, the earlier the sudden acceleration of the strain rate occurs. This strong acceleration marks the beginning of the localized neck [19]. The strengthening process results in important grain size reduction of the surface layer changing from a few micrometres down to a few nanometres. So, the grain size seems to influence significantly the onset of the strain rate heterogeneity.

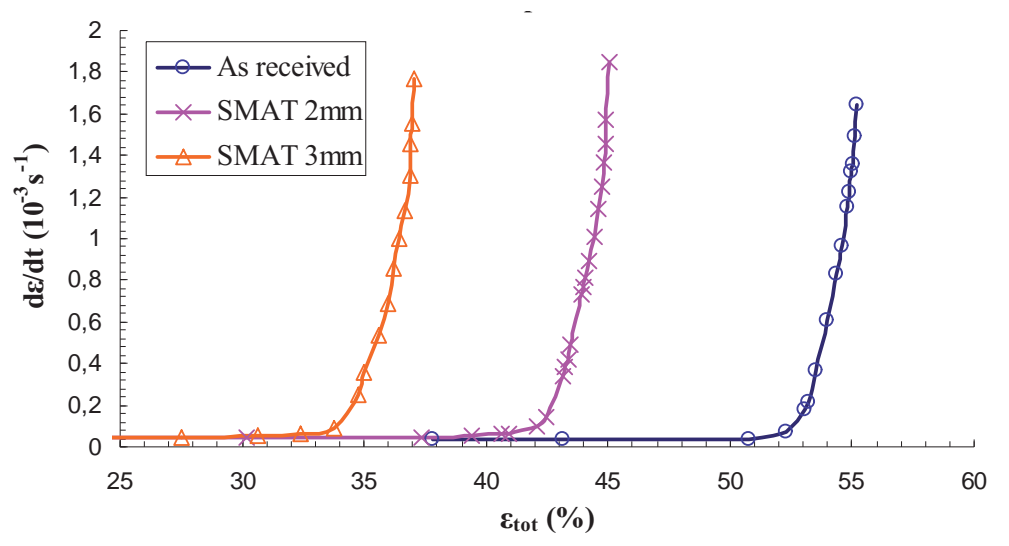

Fig. 6. Strain rate comparison in central zone of each specimen.

It is also interesting to note on Figure 6 that, for the three specimens, the strain rate present roughly the same evolution. It can also be seen on Figure 5, that there is about $7 \%$ of total strain between the maximal force and the rupture for each specimen. Moreover, it can be noticed for the three curves Figure 6, there is approximately 3,5\% of total strain between the localized neck and the rupture. Therefore, although the SMAT has an important influence on the maximal tensile force and the start of the localization, it does not affect the deformation capacity of the material after the diffuse neck appears.

To confirm this assertion, an exponential function, with $A, B$ and $C$ the parameters to settle, was fitted on each curve:

$\left.f\left(\varepsilon_{\text {tot }}\right)=\operatorname{Exp} A\left(\varepsilon_{\text {tot }}-B\right)\right\rfloor+C$,

The results were reported in Table 1 . The parameter $A$, mainly leading the growth of the exponential function, is nearly the same for the three specimens. The difference between the three values of $A$ is less than measurement uncertainties. The parameter $B$ roughly gives the start of the sudden acceleration. $B$ is consistent with tensile curves. The parameter $C$ theoretically represents the normalized global strain rate related to the reference tensile speed $v_{0}$ of $1 \mu \mathrm{m} / \mathrm{s}$ and which should be equal to approximately $0,05 \mathrm{~s}^{-1}$ (with an initial specimen length of $19 \mathrm{~mm}$ ). This is well enough verified for the two SMATed specimens, but not for the as received one. However, this last parameter plays a minor part in modelization of the strain rate evolution.

\begin{tabular}{|c|c|c|c|}
\hline Parameters & SMAT 3mm & SMAT 2mm & As received \\
\hline$A$ & 0,856 & 0,863 & 0,871 \\
\hline$B$ & 36,491 & 44,441 & 54,627 \\
\hline$C$ & 0,041 & 0,035 & $-0,009$ \\
\hline
\end{tabular}

Tab. 1. Exponential function parameters modelizing the strain rate evolution of the three $316 \mathrm{~L}$ stainless steel specimens. 


\section{Conclusion}

In this paper, comparison of strain rates, using ESPI, of as received and SMATed (Surface Mechanical Attrition Treatment) 316L stainless steel has been investigated. It was shown that SMAT process has a significant influence on the maximal force and the onset of localization. Indeed, the more severely treated the material, the higher the tensile strength and the earlier the onset of strain rate heterogeneity, and consequently the earlier the rupture. It was also shown that the SMAT parameters (shot diameter) greatly affect the onset of the localization. Besides, from the point when the strain rate increases rapidly until the rupture of the material, the strain rate evolution is almost the same for the three specimens. Approximately 3.5\% of total strain separates these two points for each specimen. The refinement of the structure therefore seems to have a little influence on the way which the materials $316 \mathrm{~L}$ stainless steel is damaged.

\section{References}

1. H. Pelletier, D. Muller, P. Mille, A. Cornet, JJ. Grob, Surface \& Coatings Technology 151, 377 (2002)

2. J. Rawers, F. Crogdon, R. Krabbe, N. Duttlinger, Powder Metallurgy 39, 125 (1996)

3. H. Fujiwara, K. Ameyama, Materials Science Forum 304, 47 (1999)

4. I. Ücok, T. Ando, NJ. Grant, Materials Science \& Engineering A 133, 284 (1991)

5. N.A. Krasilnikov, Z. Pakiela, W. Lojkowski, R.Z. Valiev, Diffusion \& Defect Data Pt.B: Solid State Phenomena 94, 63 (2003)

6. K. Lu, J. Lu, Journal of Materials Science \& Technology 15, 193 (1999)

7. K. Lu, J. Lu, Materials Science \& Engineering A 375, 38 (2004)

8. WP. Tong, NR. Tao, ZB. Wang, J. Lu, K. Lu, Science 299, 686 (2003)

9. NR. Tao, ZB. Wang, WP. Tong, ML. Sui, J. Lu, K. Lu, Acta Materiala 50, 4603 (2002)

10. L. Waltz, D. Retraint, A. Roos, P. Olier, Scripta Materiala 60, 21 (2009)

11. A. Considère, Annales des Ponts et Chaussées 9, 574 (1885)

12. R. Jones, C. Wykes, Holographic and Speckle Interferometry (vol. 6, Cambridge Studies in Modern Optics, second ed., Cambridge University Press, Cambridge, 1989)

13. G. Cloud, Optical Methods of Engineering Analysis (Cambridge University Press, NY, 1995)

14. B. Guelorget, M. Francois, C. Vial-Edwards, G. Montay, Materials Science \& Engineering A 415, 234 (2006)

15. F. Labbé, Optics \& Lasers in Engineering 45, 827 (2007)

16. R. Shabadi, S. Kumar, HJ. Roven, ES. Dwarakadasa, Materials Science \& Engineering A 364, 140 (2004)

17. K. Creath, Progress in Optics 26, 349 (1988)

18. X.H. Chen, J. Lu, L. Lu, K. Lu, Scripta Materiala 52, 1039 (2005)

19. J. Petit, G. Montay, M. Francois, Mater. Sci. Forum, 614, 155 (2008)

20. G. Montay, M. Francois, M. Tourneix, C. Vial-Edwards, I. Lira, Opt. \& Lasers Eng., 45, 222 (2007) 